\title{
THE SECOND VARIATION OF THE DIRICHLET ENERGY ON CONTACT MANIFOLDS
}

\author{
By Shangrong Deng
}

\section{Introduction}

S.S. Chern and R.S. Hamilton in a paper of 1985 [5] studied a kind of Dirichlet energy in terms of the torsion $\tau\left(\tau=\mathcal{L}_{\xi} g\right)$ of a 3-dimensional compact contact manifold and a problem analogous to the Yamabe problem. They raised the question of determining all 3-dimensional contact manifolds with $\tau=0$ (i.e. K-contact). In a long paper of 1989 [8] S. Tanno studied the Dirichlet energy and gauge transformations of contact manifolds. D.E. Blair [2] obtained the critical point condition of $I(g)=\int_{M} R i c(\xi) d V_{g}$ over $\mathscr{M}(\eta)$ (the space of all the associated metrics), and proved that the regularity of the characteristic vector field $\xi$ and the critical point condition force the metric to be K-contact. Since $\operatorname{Ric}(\xi)=2 n-1 / 4|\tau|^{2}$, the study of $I(g)$ is the same as the study of the Dirichlet energy. In this paper we investigate the second variation and prove the following result.

THEOREM 2. Let $M^{2 n+1}$ be a compact contact manifold. If $g$ is a critical metric of the Dirichlet energy $L(g)=\int_{M}|\tau|^{2} d V_{g}$, i.e. $\nabla_{\xi} L_{\xi} g=2\left(\mathcal{L}_{\xi} g\right) \phi$, then along any path $g_{\imath j}(t)=g_{\imath r}\left[\delta_{j}^{r}+t H_{j}^{r}+t^{2} K_{j}^{r}+O\left(t^{3}\right)\right]$ in $\mathscr{M}(\eta)$

$$
\frac{d^{2} L}{d t^{2}}(0)=2 \int_{M}\left|\mathcal{L}_{\xi} H_{j}^{i}\right|^{2} d V_{g} \geqq 0,
$$

and $L(g)$ has minimum at each critical metric.

The author would like to thank Professor David E. Blair for his constant encouragement and help.

\section{Contact manifolds}

A $C^{\infty}$ manifold $M^{2 n+1}$ is said to be a contact manifold if it carries a global 1-form $\eta$ such that $\eta \wedge(d \eta)^{n} \neq 0$ everywhere. Given a contact form $\eta$ it is well

Received February 5, 1991; revised April 15, 1991. 
known that there exists a unique vector field $\xi$ on $M$ satisfying $d \eta(\xi, X)=0$ and $\eta(\xi)=1 ; \xi$ is called the characteristic vector field of the contact structure. A Riemannian metric $g$ is said to be an associated metric if there exists a tensor field $\phi$ of type $(1,1)$ such that $d \eta(X, Y)=g(X, \phi Y), \phi^{2}=-I+\eta \otimes \xi$ and $\eta(X)=$ $g(X, \xi)$. We call $(\phi, \xi, \eta, g)$ a contact metric structure. Such $\phi$ and $g$ can be constructed by the polarization of $d \eta$ and they are not unique (see [4]). All associated metrics have the same volume element, namely $d V=\left(1 / 2^{n} n !\right) \eta \wedge d \eta^{n}$.

Let $\tau=\mathcal{L}_{\xi} g$ be the torsion and let $h=(1 / 2) \mathcal{L}_{\xi} \phi$. We have

$$
\begin{aligned}
& \tau_{\imath \jmath}=-2 \phi_{i r} h_{\jmath}^{r} \\
& h_{r}^{\imath} \phi_{j}^{r}+\phi_{r}^{\imath} h_{\jmath}^{r}=0 \\
& \nabla_{\imath} \eta_{\jmath}=\phi_{i j}-\phi_{i r} h_{\jmath}^{r}
\end{aligned}
$$

where $\phi_{i \jmath}=g_{\imath r} \phi_{j}^{r}$, and

$$
\operatorname{Ric}(\xi)=2 n-|h|^{2}=2 n-\frac{1}{4}|\tau|^{2} .
$$

We call the contact metric structure with $\tau=h=0$ (or $\xi$ is Killing) K-contact. For general reference see [1], [7] and [9].

\section{The space of all associated metrics and the Dirichlet energy}

The space of all Riemannian metrics of $M^{2 n+1}$ with fixed volume, denoted by $\mathscr{M}_{1}$, is a symmetric Hilbert manifold; geodesics in $\mathscr{M}_{1}$ are of the form $g e^{H t}$ (here $H$ is a type $(1,1)$ tensor field; see $[6])$. The space of all the associated metrics $\mathscr{M}(\eta)$ is a totally geodesic submanifold of $\mathscr{M}_{1}$. See [4] for details about $\mathscr{M}(\eta)$. Let $g(t)$ be any curve in $\mathscr{M}(\eta)$ with $g(0)=g$. Then the structure tensors $(\phi(t), \xi, \eta, g(t))$ corresponding to $g(t)$ satisfy the following:

$$
\begin{aligned}
& g_{\imath r}(t) \xi^{r}=\eta_{\imath} \\
& 2 g_{\imath r}(t) \phi_{j}^{r}(t)=2 \phi_{i j}=\nabla_{\imath} \eta_{j}-\nabla_{j} \eta_{\imath} \\
& \phi_{r}^{2}(t) \phi_{j}^{r}(t)=-\delta_{j}^{2}+\xi^{\imath} \eta_{\jmath} .
\end{aligned}
$$

Now we put

$$
\begin{aligned}
& g_{\imath j}(t)=g_{\imath r}\left[\delta_{j}^{r}+t H_{j}^{r}+t^{2} K_{j}^{r}+O\left(t^{3}\right)\right] \\
& \phi_{j}^{\imath}(t)=\phi_{j}^{2}+t S_{j}^{i}+t^{2} T_{j}^{i}+O\left(t^{3}\right) .
\end{aligned}
$$

Then from the above conditions we have

$$
\begin{aligned}
& H_{\imath r} \xi^{r}=K_{\imath r} \xi^{r}=S_{r}^{i} \xi^{r}=T_{r}^{i} \xi^{r}=0 \\
& H_{\imath j}+H_{r s} \phi_{i}^{r} \phi_{\jmath}^{s}=0, \quad \text { hence } H_{i}^{i}=0 \\
& S_{\jmath}^{i}=\phi_{r}^{\imath} H_{\jmath}^{r}, \quad S_{r}^{i} S_{\jmath}^{r}=H_{r}^{i} H_{\jmath}^{r} \\
& T_{\jmath}^{i}=\phi_{r}^{\imath} K_{\jmath}^{r}
\end{aligned}
$$




$$
\begin{aligned}
& K_{\imath j}+K_{r s} \phi_{i}^{r} \phi_{j}^{s}=H_{\imath r} H_{j}^{r} \\
& 2 K_{r}^{r}=H^{r s} H_{r s}
\end{aligned}
$$

where $H_{\imath \jmath}=g_{\imath r} H_{\jmath}^{r}$, etc., and the inverse of $g(t)$ is given by ([8])

$$
g^{i j}(t)=g^{i j}-t H^{i j}+t^{2}\left(H_{r}^{i} H^{r j}-K^{i j}\right)+O\left(t^{3}\right) .
$$

The critical point condition of the Dirichlet energy $L(g)=\int_{M}|\tau|^{2} d V_{g}$ is given by the following theorem, see [2], [5] and [8] for proof.

THEOREM 1. Let $M^{2 n+1}$ be a compact contact manifold. An associated metric $g \in \mathscr{M}(\eta)$ is critical with respect to the Dirichlet energy if and only if

$$
\nabla_{\xi} \tau=2 \tau \phi \text {. }
$$

Remarks. Chern and Hamilton studied this over the set of all the $C R$ structures. Strongly pseudo-convex $C R$-manifolds are contact manifolds satisfying an integrability condition i.e. $Q=0$; in dimension $3 Q=0$ trivially (see [8]).

\section{Proof of Theorem 2}

THEOREM 2. Let $M^{2 n+1}$ be a compact contact manifold. If $g$ is a critical metric of the Dirichlet energy, i.e. $\nabla_{\xi} \tau=2 \tau \phi$, then along any path $g(t)$ in $\mathscr{M}(\eta)$ with $g(0)=g$

$$
\frac{d^{2} L}{d t^{2}}(0)=2 \int_{M}\left|\mathcal{L}_{\xi} H_{j}^{i}\right|^{2} d V_{g} \geqq 0,
$$

and $L(g)$ has minimum at each critical metric.

Proof. Let $g_{\imath j}(t)=g_{i j}+t H_{\imath j}+t^{2} K_{\imath j}+O\left(t^{3}\right)$ be any curve in $\mathscr{M}(\eta)$ with $g(0)=g$ critical. Then for the curvature tensor we have

$$
\begin{aligned}
R_{i j k}{ }^{h}(t)= & R_{i j k}{ }^{h}+\frac{t}{2}\left(\nabla_{\imath} D_{j k}{ }^{h}-\nabla_{j} D_{i k}{ }^{n}\right) \\
& +\frac{t^{2}}{2}\left[\nabla_{i}\left(E_{j k}{ }^{h}-H_{r}^{h} D_{j k}{ }^{r}\right)-\nabla_{j}\left(E_{i k}{ }^{n}-H_{r}^{h} D_{i k}{ }^{r}\right)\right. \\
& \left.+\frac{1}{2}\left(D_{i r}{ }^{h} D_{j k}{ }^{r}-D_{\jmath r}{ }^{h} D_{i k}{ }^{r}\right)\right]+O\left(t^{3}\right)
\end{aligned}
$$

where $D_{j k}{ }^{2}=\nabla_{j} H_{k}^{i}+\nabla_{k} H_{j}^{i}-\nabla^{i} H_{j k}, E_{j k}{ }^{2}=\nabla_{j} K_{k}^{i}+\nabla_{k} K_{j}^{i}-\nabla^{i} K_{j k}$. Therefore we have

$$
\begin{aligned}
R_{j k}(t)= & R_{j k}+\frac{t}{2}\left(\nabla_{r} \nabla_{j} H_{k}^{r}+\nabla_{r} \nabla_{k} H_{j}^{r}-\nabla^{r} \nabla_{r} H_{j k}\right) \\
& +\frac{t^{2}}{4}\left[2\left(\nabla_{r} \nabla_{j} K_{k}^{r}+\nabla_{r} \nabla_{k} K_{j}^{r}-\nabla^{r} \nabla_{r} K_{j k}-\nabla_{j} \nabla_{k} K_{r}^{r}\right)\right.
\end{aligned}
$$




$$
\begin{aligned}
& -2 H^{r s}\left(\nabla_{s} \nabla_{j} H_{r k}+\nabla_{s} \nabla_{k} H_{r j}-\nabla_{s} \nabla_{r} H_{j k}-\nabla_{j} \nabla_{k} H_{r s}\right) \\
& -2 \nabla_{s} H^{s r}\left(\nabla_{j} H_{r k}+\nabla_{k} H_{r j}-\nabla_{r} H_{j k}\right) \\
& \left.+\nabla_{j} H^{r s} \nabla_{k} H_{r s}-2 \nabla_{r} H_{j}^{s} \nabla_{s} H_{k}^{r}+2 \nabla_{r} H_{j}^{s} \nabla^{r} H_{s k}\right]+O\left(t^{3}\right) .
\end{aligned}
$$

See [8] for some details. Let $I(g)=\int_{M} \operatorname{Ric}(\xi) d V_{g}$. For any associated metric we have $\operatorname{Ric}(\xi)=2 n-(1 / 4)|\tau|^{2}$, hence $I(g)=2 n \operatorname{vol}(M)-(1 / 4) L(g)$. Now we assume

$$
\begin{aligned}
I_{1}= & \int_{M} \xi^{j} \xi^{l}\left(\nabla_{r} \nabla_{l} K_{j}^{r}+\nabla_{r} \nabla_{j} K_{l}^{r}-\nabla_{r} \nabla^{r} K_{j l}-\nabla_{l} \nabla_{j} K_{r}^{r}\right) d V_{g} \\
I_{2}= & \int_{M} \xi^{\jmath} \xi^{l}\left[-H^{r s}\left(\nabla_{r} \nabla_{l} H_{s j}+\nabla_{r} \nabla_{j} H_{s l}-\nabla_{r} \nabla_{s} H_{j l}-\nabla_{l} \nabla_{j} H_{r s}\right)\right. \\
& -\nabla_{s} H^{s r}\left(\nabla_{l} H_{r j}+\nabla_{j} H_{r l}-\nabla_{r} H_{j l}\right)+\frac{1}{2} \nabla_{l} H^{r s} \nabla_{j} H_{r s} \\
& \left.+\nabla_{r} H_{s j} \nabla^{r} H_{l}^{s}-\nabla_{r} H_{s j} \nabla^{s} H_{l}^{r}\right] d V_{g} .
\end{aligned}
$$

Then for $I(g)$ we have

$$
\frac{d^{2} I}{d t^{2}}(0)=I_{1}+I_{2}
$$

Using Green's Theorem, the critical point condition and the facts that

$$
\begin{aligned}
& H_{r}^{i} H_{s}^{r} h_{\imath}^{s}=\nabla_{\xi} H_{s}^{r} H_{i}^{s} h_{j}^{2} \phi_{r}^{\jmath}=0 \\
& \nabla^{r} \xi^{i} \nabla_{i} \xi^{s}=-g^{r s}+\xi^{r} \xi^{s}+h_{j}^{r} h^{\jmath s} \\
& \nabla^{i} \xi^{r} \nabla_{i} \xi^{s}=g^{r s}-\xi^{r} \xi^{s}-2 h^{r s}+h_{j}^{r} h^{\jmath s}
\end{aligned}
$$

we compute as follows

$$
\begin{aligned}
& \int_{M} \xi^{\jmath} \xi^{l} \nabla_{r} \nabla_{l} K_{j}^{r} d V_{g}=\int_{M}\left(\xi^{l} \nabla_{l} \nabla^{r} \xi^{s}+\nabla_{l} \xi^{s} \nabla^{r} \xi^{l}\right) K_{r s} d V_{g} \\
& \int_{M} \xi^{\jmath} \xi^{l} \nabla_{r} \nabla^{r} K_{j l} d V_{g}=2 \int_{M} \nabla_{r} \xi^{j} \nabla^{r} \xi^{l} K_{j l} d V_{g} \\
& \int_{M} \xi^{\jmath} \xi^{l} \nabla_{l} \nabla_{j} K_{r}^{r} d V_{g}=0
\end{aligned}
$$

and hence

$$
\begin{aligned}
I_{1} & =2 \int_{M}\left(\xi^{l} \nabla_{l} \nabla^{r} \xi^{s}+\nabla_{l} \xi^{s} \nabla^{r} \xi^{l}-V_{i} \xi^{s} \nabla^{i} \xi^{r}\right) K_{r s} d V_{g} \\
& =-4 \int_{M} K_{r}^{r} d V_{g} \\
& =-2 \int_{M}|H|^{2} d V_{g} .
\end{aligned}
$$


Now consider $I_{2}$

$$
\begin{aligned}
& \int_{M} \xi^{\jmath} \xi^{l} H^{r s} \nabla_{r} \nabla_{l} H_{s j} d V_{g}= \int_{M}\left[\nabla_{l} \nabla_{r} \xi^{\jmath} \xi^{l} H^{r s} H_{s j}+\nabla_{r} \xi^{\jmath} \xi^{l} \nabla_{l} H^{r s} H_{s \jmath}\right. \\
&\left.+\nabla_{l} \xi^{j} \nabla_{r} \xi^{l} H^{r s} H_{s j}-\xi^{\jmath} \xi^{l} \nabla_{r} H^{r s} \nabla_{l} H_{s \jmath}\right] d V_{g} \\
& \int_{M} \xi^{\jmath} \xi^{l} H^{r s} \nabla_{r} \nabla_{s} H_{j l} d V_{g}= \int_{M}\left[2 \nabla_{r} \xi^{j} \nabla_{s} \xi^{l} H^{r s} H_{j l}-\xi^{\jmath} \xi^{l} \nabla_{r} H^{r s} \nabla_{s} H_{j l}\right] d V_{g} \\
& \int_{M} \xi^{\jmath} \xi^{l} H^{r s} \nabla_{l} \nabla_{j} H_{r s} d V_{g}=-\int_{M}\left|\nabla_{\xi} H\right|^{2} d V_{g} \\
& \int_{M} \xi^{\jmath} \xi^{l} \nabla_{r} H_{s j} \nabla^{r} H_{l}^{s} d V_{g}=\int_{M} \nabla_{r} \xi^{j} \nabla^{r} \xi^{l} H_{s j} H_{l}^{s} d V_{g} \\
& \int_{M} \xi^{\jmath} \xi^{l} \nabla_{r} H_{s j} \nabla^{s} H_{l}^{r} d V_{g}=\int_{M} \nabla_{r} \xi^{j} \nabla^{s} \xi^{l} H_{s j} H_{l}^{r} d V_{g} .
\end{aligned}
$$

Therefore

$$
\begin{aligned}
I_{2}= & \int_{M}\left[-2 \nabla_{l} \nabla_{r} \xi^{\jmath} \xi^{l} H^{r s} H_{s j}-2 \nabla_{r} \xi^{\jmath} \xi^{l} \nabla_{l} H^{r s} H_{s \jmath}\right. \\
& -2 \nabla_{l} \xi^{j} \nabla_{r} \xi^{l} H^{r s} H_{s j}+2 \nabla_{r} \xi^{j} \nabla_{s} \xi^{l} H^{r s} H_{j l} \\
& +\nabla_{r} \xi^{j} \nabla^{r} \xi^{l} H_{\jmath s} H_{l}^{s}-\nabla_{r} \xi^{\jmath} \nabla^{s} \xi^{l} H_{s j} H_{l}^{r} \\
& \left.-\frac{1}{2}\left|\nabla_{\xi} H\right|^{2}\right] d V_{g}
\end{aligned}
$$

but

$$
\begin{aligned}
& \int_{M} \xi^{l} \nabla_{l} \nabla_{r} \xi^{\jmath} H^{r s} H_{s j} d V_{g}=0 \\
& \int_{M} \nabla_{l} \xi^{j} \nabla_{r} \xi^{l} H^{r s} H_{s j} d V_{g}=\int_{M}\left(-|H|^{2}+|h H|^{2}\right) d V_{g} \\
& \int_{M} \nabla_{r} \xi^{j} \nabla_{s} \xi^{l} H^{r s} H_{j l} d V_{g}=\int_{M}\left(-|H|^{2}-\operatorname{tr}(h H)^{2}\right) d V_{g} \\
& \int_{M} \nabla_{r} \xi^{j} \nabla^{r} \xi^{l} H_{s j} H_{l}^{s} d V_{g}=\int_{M}\left(|H|^{2}+|h H|^{2}\right) d V_{g} \\
& \int_{M} \nabla_{r} \xi^{j} \nabla^{s} \xi^{l} H_{s j} H_{l}^{r} d V_{g}=\int_{M}\left(|H|^{2}-\operatorname{tr}(h H)^{2}\right) d V_{g}
\end{aligned}
$$

and hence

$$
\begin{aligned}
I_{2}= & \int_{M}\left[2\left(\phi_{r}^{j}+\phi_{r i} h^{\imath \jmath}\right) \nabla_{\xi} H_{s}^{r} H_{j}^{s}-t r(h H)^{2}\right. \\
& \left.-|h H|^{2}-\frac{1}{2}\left|\nabla_{\xi} H\right|^{2}\right] d V_{g} .
\end{aligned}
$$

Since $\phi_{r i} h^{2 j} \nabla_{\xi} H_{s}^{r} H_{j}^{s}=0$, we have 


$$
\begin{aligned}
\frac{d^{2} I}{d t^{2}}(0)= & I_{1}+I_{2} \\
= & \int_{M}\left[-2|H|^{2}+2 \phi_{r}^{j} \nabla_{\xi} H_{s}^{r} H_{j}^{s}-\frac{1}{2}\left|\nabla_{\xi} H\right|^{2}\right. \\
& \left.-\operatorname{tr}(h H)^{2}-|h H|^{2}\right] d V_{g} \\
= & \int_{M}\left[-\frac{1}{2}\left|2 H-\phi \nabla_{\xi} H\right|^{2}-\operatorname{tr}(h H)^{2}-|h H|^{2}\right] d V_{g} .
\end{aligned}
$$

Now note that

$$
\begin{aligned}
\left|\mathcal{L}_{\xi} H_{j}^{i}\right|^{2} & =\left|\nabla_{\xi} H-2 H \phi\right|^{2}+|H h+h H|^{2} \\
& =\left|\nabla_{\xi} H-2 H \phi\right|^{2}+2 \operatorname{tr}(h H)^{2}+2|h H|^{2}
\end{aligned}
$$

therefore

$$
\frac{d^{2} L}{d t^{2}}(0)=(-4) \frac{d^{2} I}{d t^{2}}(0)=2 \int_{M}\left|\mathcal{L}_{\xi} H_{j}^{i}\right|^{2} d V_{g} \geqq 0 .
$$

We show in the next proposition that $|\tau(t)|^{2}$ is constant along any geodesic $g(t)=g e^{H t}$ with $\mathcal{L}_{\xi} H_{j}^{i}=0$, hence, $L(g)$ is constant along all such geodesics. $\mathscr{M}(\eta)$ is geodesically complete [4], therefore $L(g)$ has minimum at each critical metric.

Q.E.D.

Proposition. $\quad \tau_{j}^{\imath}(t)=\tau_{j}^{\imath}(0)$ along any geodesic $g(t)=g e^{H t}$ with $\mathcal{L}_{\xi} H_{j}^{i}=0 . \quad$ In particular, $|\tau(t)|^{2}$ is constant along such geodesics.

Proof. Let $D_{j k}^{(n) \imath}=\nabla_{j}\left(H^{n}\right)_{k}^{\imath}+\nabla_{k}\left(H^{n}\right)_{j}^{\imath}-\nabla^{i}\left(H^{n}\right)_{j k}$. If $\mathcal{L}_{\xi} H_{j}^{i}=0$, we have $\nabla_{\xi} H$ $=2 H \phi$ and $h H=-H h$, and hence

$$
\begin{aligned}
D_{j k}^{(n) \imath} \xi^{k} & =\nabla_{\xi}\left(H^{n}\right)_{j}^{\imath}+\left(H^{n}\right)_{k}^{\imath} \phi_{j}^{k}+\phi_{k}^{\imath}\left(H^{n}\right)_{j}^{k}-\left(H^{n}\right)_{r}^{\imath} h_{k}^{r} \phi_{j}^{k}-\phi_{r}^{\imath} h_{k}^{r}\left(H^{n}\right)_{j}^{k} \\
& =2\left(H^{n}\right)_{r}^{\imath} \phi_{j}^{r}
\end{aligned}
$$

for any $n$. Thus along $g e^{H t}$ with $\mathcal{L}_{\xi} H_{\jmath}^{i}=0$,

$$
\begin{aligned}
\nabla_{j}^{(t)} \xi^{\imath} & =\nabla_{j} \xi^{2}+\frac{t}{2} D_{j k}{ }^{2} \xi^{k}+\frac{t^{2}}{2}\left(\frac{1}{2} D_{j k}^{(2) \imath} \xi^{k}-H_{r}^{i} D_{j k}{ }^{2} \xi^{k}\right)+\cdots \\
& +\frac{t^{n}}{2}\left[\frac{1}{n !} D_{j k}^{(n) \imath}+\frac{1}{(n-1) !}(-1) H_{r}^{i} D_{j k}^{(n-1) r}+\frac{1}{(n-2) ! 2 !}\left(H^{2}\right)_{r}^{\imath} D_{j k}^{(n-2) r}+\cdots\right. \\
& \left.+\frac{1}{(n-l) ! l !}(-1)^{l}\left(H^{l}\right)_{r}^{\imath} D_{j k}^{(n-l) r}+\cdots+\frac{1}{(n-1) !}(-1)^{n-1}\left(H^{n-1}\right)_{r}^{\imath} D_{j k}{ }^{r}\right] \xi^{k} \\
& +\cdots
\end{aligned}
$$

and therefore

$$
-\phi_{j}^{2}(t)+\frac{1}{2} \tau_{j}^{2}(t)=-\phi_{j}^{2}+\frac{1}{2} \tau_{j}^{2}-t \phi_{r}^{2} H_{j}^{r}-\cdots-\frac{t^{n}}{n !} \phi_{r}^{2}\left(H^{n}\right)_{j}^{r}-\cdots .
$$


Note that $\phi(t)=\phi e^{H t}$; therefore we have

$$
\tau_{j}^{\imath}(t)=\tau_{j}^{\imath}(0)
$$

along $g e^{H t}$ with $\mathcal{L}_{\xi} H_{j}^{i}=0$.

Q.E.D.

Example 1. Any $K$-contact manifold, since $\tau=0, L(g)$ has minimum trivially.

Example 2. The tangent sphere bundle of a compact Riemannian manifold of constant curvature $(-1)$, i.e. $T_{1} M(-1)$ (see [3]). In this case the standard associated metric is a critical point of $L(g)$, but $\tau$ is not 0 . In fact, non-trivial examples must be irregular (see [2]). Theorem 2 says that $L(g)$ has local minimum at the standard metric. It seems that it is also a global minimum, or in other words, one can not deform the metric to have $\tau=0$.

\title{
REFERENCES
}

[1] D. E. Blair, Contact Manifolds in Riemannian Geometry, Lect. Notes in Math. Vol. 509, Springer, Berlin.

[2] D.E. Blair, Critical associated metrics on contact manifolds, J. Aust. Math. Soc. 37 (1984), 82-88.

[3] D. E. BlaIR, Critical associated metrics on contact manifolds III. preprint.

[4] D.E. BLAIR, On the set of metrics associated to a sympletic or contact form, Bull. Inst. Math. Acad. Sinica. 11 (1983), 297-308.

[5] S.S. Chern and R.S. Hamilton, On Riemannian metrics adapted to threedimensional contact manifolds, Lect. Notes in Math. Vol. 1111, Springer, Berlin, 279-305.

[6] D. Ebin, The manifold of Riemannian metrics, Proc. Symp. Pure Math. AMS. 15 (1970), 11-40.

[7] S. SASAKI, Almost Contact Manifolds, Lecture Notes. Tôhoku Univ. Vol. 1 1965, Vol. 2 1967, Vol. 31968.

[8] S. Tanno, Variational problems on contact Riemannian manifolds, Trans. Amer. Math. Soc. 314 (1989), 349-379.

[9] K. Yano And M. Kon, Structures on Manifolds, World Scientific. 1984.

\author{
Mathematics \\ SOUTHERN TECH. \\ MARIETTA, GA 30060 \\ U.S. A.
}

